\title{
MONOTONE SUBNETS IN PARTIALLY ORDERED SETS
}

R. W. HANSELL

1. Introduction. Let $X$ be a partially ordered set (poset) with respect to a relation $\geqq$. We assume, for convenience of notation only, that $X$ has a greatest element $I$ and a least element $O$. A net $(f, D, \succ)$ in $X$ is monotone increasing (decreasing) iff whenever $\alpha \succ \beta$ in $D$, then $f(\alpha) \geqq f(\beta)(f(\beta) \geqq f(\alpha))$ in $X$. A net is said to be monotone if it is either monotone increasing or monotone decreasing. It is the purpose of this paper to characterize those posets $X$ for which every net in $X$ has a monotone subnet, and to derive some of their properties. It is shown that every net in $X$ possesses a monotone subnet iff $X$ contains no infinite totally unordered subset. This is accomplished mainly through the use of universal nets (our terminology and notation for nets are those of Kelley [1]). The theorem is a generalization of the well-known result of analysis which states that every sequence of real numbers contains a monotone subsequence. Our results are then applied to obtain a simple proof of a known theorem on unique order-compatible topologies for posets (see [6], [7]). Also, it is shown, that for the same class of posets, one obtains a generalization of the well-known theorem of Frink's, which states that a lattice is complete iff it is compact in its interval topology.

2. Principal results. Let us call a net $(f, D, \succ)$ in $X$ diverse iff whenever $\alpha \succ \beta$ and $\alpha \neq \beta$ in $D$, then $f(\alpha)$ and $f(\beta)$ are incomparable in $X$. The following theorem will be fundamental in establishing our principal result.

Theorem 1. Every universal net in a poset $X$ possesses a monotone subnet or a diverse subnet.

Proof. Let $(u, D, \succ)$ be a universal net in $X$. For each $\alpha$ in $D$ we decompose $X$ in to three mutually disjoint sets as follows:

$$
\begin{aligned}
& U_{\alpha}=\{x \in X \mid x>u(\alpha)\}, \\
& L_{\alpha}=\{x \in X \mid x \leqq u(\alpha)\}, \text { and } \\
& N_{\alpha}=\{x \in X \mid x \text { and } u(\alpha) \text { are incomparable }\} .
\end{aligned}
$$

Since $u$ is universal, it follows that for each $\alpha$ in $D u$ is eventually in one of the above three sets. Accordingly, we decompose $D$ into the sets

Received by the editors September 10, 1966. 


$$
\begin{aligned}
& U=\left\{\alpha \in D \mid u \text { is eventually in } U_{\alpha}\right\}, \\
& L=\left\{\alpha \in D \mid u \text { is eventually in } L_{\alpha}\right\}, \text { and } \\
& N=\left\{\alpha \in D \mid u \text { is eventually in } N_{\alpha}\right\} .
\end{aligned}
$$

Since this is, in fact, a decomposition of $D$, one of the sets $U, L$, or $N$ is cofinal in $D$. We now assert that according as $U, L$, or $N$ is cofinal, $u$ possesses an increasing, decreasing, or diverse subnet.

First observe that each of the three cases can be described in the following general manner: there exists a cofinal subset $E$ of $D$ such that to each $\alpha$ in $E$ there is associated a set $X_{\alpha} \subseteq X$ which eventually contains the range of $u$. We now show that under these conditions there exists a subnet of $u$ of the form $\left(u, E, \succ^{\prime}\right)$ and with the property that whenever $\alpha \succ^{\prime} \beta$ and $\alpha \neq \beta$, then $u(\alpha) \in X_{\beta}$. One sees easily that this is the desired result when given the interpretations above.

Since $u$ is eventually in $X_{\beta}$ for each $\beta$ in $E$, we may choose $i_{\beta}>\beta$ in $D$ such that $\alpha \succ i_{\beta}$ implies $u(\alpha) \in X_{\beta}$. Thus if $\alpha$ and $\beta$ are arbitrary members of $E$, define $\alpha \succ^{\prime} \beta$ iff $\alpha=\beta$ or $\alpha \succ^{\prime} i_{\beta}$. Evidently, $\succ^{\prime}$ is a reflexive and transitive relation in $E$. Furthermore, since the identity mapping from $\left(E, \succ^{\prime}\right)$ to $(D, \succ)$ is isotone and $E$ is cofinal in $D$, we need only to prove that $\succ$ directs $E$ (cf. [1, p. 70]). But if $\alpha$ and $\beta$ are any members of $E$ there exists $\gamma$ in $E$ such that $\gamma \succ$ both $i_{\alpha}$ and $i_{\beta}$, since $E$ is cofinal in $D$. Hence $\gamma \succ^{\prime} \alpha$ and $\gamma \succ^{\prime} \beta$, completing the proof.

Our main theorem will follow as a consequence of Theorem 1 and the following lemma.

Lemma. Let $(f, D, \succ)$ be a diverse net in $X$ and suppose that $(D, \succ)$ has no greatest member. Then there exists an infinite totally unordered subset of $X$ contained in the range of $f$.

Proof. Select $\alpha_{1} \in D$ arbitrarily. We shall construct inductively the desired subset of $X$. Thus assume we have chosen for $i=1, \cdots, k-1$, elements $\alpha_{i} \in D$ such that $\left\{f\left(\alpha_{i}\right) \mid i=1, \cdots, k-1\right\}$ is a totally unordered subset of $X$. Now choose $\alpha_{k} \in D$ such that for all $i$ we have $\alpha_{k} \succ \alpha_{i}, \alpha_{k} \neq \alpha_{i}$. This is possible since $(D, \succ)$ has no greatest member. Moreover, since $f$ is diverse, $f\left(\alpha_{k}\right)$ is incomparable with each $f\left(\alpha_{i}\right)$. It follows by induction that range $(f)$ contains an infinite totally unordered subset.

We now have the following theorem.

Theorem 2. Every net in a poset $X$ possesses a monotone subnet if and only if $X$ contains no infinite totally unordered subset.

Proof. The condition is necessary, for any sequence of pairwise 
incomparable elements of $X$ is a net in $X$ which clearly contains no monotone subnet.

Since it is well known $[1$, p. 80$]$ that every net possesses a subnet which is universal, the sufficiency of the condition will follow if we can show that every universal net in $X$ has a monotone subnet. Thus let $u$ be any universal net in $X$. We may assume that the domain of $u$ has no greatest member. Then, by Theorem $1, u$ has either a monotone subnet or a diverse subnet whose domain has no greatest member. Since, by the Lemma, the latter is not possible whenever $X$ possesses no infinite totally unordered subset, it follows that every universal net in $X$ possesses a monotone subnet, and this completes the proof.

As an obvious corollary to Theorem 2 we have the following.

CoROLlary. Every net in a linearly ordered set contains a monotone subnet.

It is also worth noting that Theorem 2 remains valid if the words "sequence" and "subsequence" are substituted for the words "net" and "subnet," respectively.

3. Order-compatible topologies. A subset $S$ of $X$ is said to be $u p$ directed (down-directed) iff for all $x S \in$ and $y \in S$ there exists $z \in S$ such that $z \geqq x, z \geqq y(z \leqq x, z \leqq y)$. Following McShane [4] and Wolk [6] we call a subset $K$ of $X$ Dedekind-closed iff whenever $S$ is an updirected subset of $K$ and $y=1$.u.b. $(S)$, or $S$ is a down-directed subset of $K$ and $y=$ g.l.b. $(S)$, we have $y \in K$. Let $D$ denote the topology on $X$ whose closed sets are the Dedekind-closed subsets of $X$. We let $\mathfrak{I}$ denote the well-known interval topology on $X$, which results from taking as a subbase for the closed sets of $X$ all sets of the form $\{x \in X \mid a \leqq x \leqq b\}$. E. S. Wolk [6] has introduced and studied the following notion of an order-compatible topology on a poset, which seems to be a natural requirement for a topology on $X$ to be harmoniously related to its order structure. A topology $J$ on $X$ is said to be order-compatible iff it satisfies the relation $g \subseteq J \subseteq D$.

The main purpose of this section is to show that one of the principal results on unique order-compatible topologies for posets can be deduced rather easily from Theorem 2 . We shall have need of the following lemma, which is in essence known: part (i) is essentially known for the interval topology [1, Problem 2.F] and part (ii) is a special case of a more general result $[6$, Lemma 5$]$. We omit the routine proofs together with the obvious dual formulations.

Lemma $\left(^{*}\right)$. Let $f$ be any monotone increasing net in $X$. Then 
(i) if $y=1$.u.b. range $(f)$ exists, then $f$ D-converges to $y$, and

(ii) if $y$ is any g-cluster point of $f$, then necessarily $y=1$.u.b. range $(f)$.

Theorem (Wolk [6] AND NaIto [5]). If $X$ is a poset such that every totally unordered subset of $X$ has cardinality less than $\boldsymbol{\aleph}_{0}$, then $X$ possesses a unique order-compatible topology. Furthermore, $X$ is a Hausdorff space with respect to this topology.

Proof. Let $X$ satisfy the hypothesis, and let $K$ be a $D$-closed subset of $X$. Also, let $f$ be a net in $K$ which $g$-converges to an element $y \in X$. Then we must show that $y \in K$.

By Theorem 2, $f$ has a monotone subnet, $g$ say, which we take to be increasing (the other case being handled in the obvious dual manner). Since subnets of convergent nets converge, and to the same point, $g \mathfrak{g}$-converges to $y$. Hence, by Lemma $\left({ }^{*}\right), y=1$.u.b. range $(g)$. Moreover, since range $(g)$ is an up-directed subset of $K$ and $K$ is D-closed, we must have $y \in K$. Hence $K$ is $\mathcal{g}$-closed, and it follows that $X$ has a unique order-compatible topology. Furthermore, since the 1.u.b. of a set is unique, it follows that each net in $X$ converges to at most one point relative to this topology; that is, the space is Hausdorff.

4. Dedekind completeness. McShane [4] has introduced the following concept of "Dedekind completeness" for posets, which is a generalization of the usual concept of completeness for lattices. A poset $X$ is called Dedekind complete iff every up-directed subset of $X$ has a l.u.b. in $X$ and every down-directed subset has a g.l.b. in $X$. Dedekind completeness has been studied and characterized by Wolk [8] and Kogalovskii [3]. In view of Lemma $\left(^{*}\right)$, it is also clear that a poset $X$ is Dedekind complete iff every monotone net in $X$ converges, relative to some order-compatible topology on $X$.

It is natural to ask whether the well-known theorem of Frink, which states that a lattice is complete iff it is 9 -compact, can be extended to Dedekind completeness for posets. That such is not the case in general can be shown by the following example, due in essence to Kogalovskiı [2]. Let $X$ be the set of images of three infinite sequences $\left\{a_{i}\right\},\left\{b_{j}\right\}$, and $\left\{c_{k}\right\}$, and let $X$ be partially ordered as follows: (i) each of the sequences is a totally unordered subset of $X$, (ii) $a_{i}>b_{j}$ for $i \neq j$, and (iii) $b_{j}>c_{k}$ for $j \neq k$ (greatest and least elements may also be adjoined). Then one easily verifies that with this ordering $X$ is a Dedekind complete poset. However, $X$ is not compact in its interval topology. In fact, the family $\left\{\left[a_{i}, c_{i}\right] \mid i=1,2, \cdots\right\}$ of closed intervals has a void intersection, whereas the intersection of each finite subfamily is nonvoid. Thus we see that Dedekind com- 
pleteness does not in general imply compactness in the in terval topology. However, to determine which of the natural classes of posets have this property, would be results which are not without interest. We are able to show the following.

THEOREM 3. If $X$ is a poset having no infinite totally unordered subset, then $X$ is Dedekind complete iff it is compact in its $D$ topology.

Proof. First suppose $X$ is $D$-compact, and let $S$ be an up-directed subset of $X$. Letting $i$ denote the identity mapping on $S$, it is clear that $(i, S, \geqq)$ is a monotone increasing net in $X$ with range $(i)=S$. By compactness, $i$ has a cluster point $y \in X$, which, by (ii) of Lemma $\left({ }^{*}\right)$, must be the l.u.b. $(S)$. Hence l.u.b. $(S)$ exists, and dually for downdirected subsets of $X$. Therefore, $X$ is Dedekind complete.

Conversely, suppose $X$ is Dedekind complete and let $f$ be an arbitrary net in $X$. We shall show that $f$ has a convergent subnet. By the hypothesis and Theorem $2, f$ admits of a monotone subnet $g$, which we take to be increasing. Then range $(g)$ is an up-directed subset of $X$ and hence has a l.u.b. $y \in X$. But then, by (i) of Lemma $\left(^{*}\right), g$ must converge to $y$ with respect to the $D$ topology. Similarly if $g$ is decreasing. Therefore $X$ is $D$-compact, completing the proof.

REMARK. For the $D$ topology Theorem 3 is actually the best result obtainable, since $X$ is $D$-compact only if $X$ contains no infinite totally unordered subset (see [5, Theorem 2] for the details).

The author wishes to express his appreciation to Professor E. S. Wolk for his interest and assistance.

\section{REFERENCES}

1. J. L. Kelley, General topology, Van Nostrand, New York, 1955.

2. S. R. KogalovskiY, On a theorem of Frink, Uspehi Mat. Nauk. 19 (1964), 143145. (Russian)

3. - On linearly complete order sets, Uspehi Mat. Nauk 19 (1964), 147-150. (Russian)

4. E. J. McShane, Order-preserving maps and integration processes, Annals of Mathematics Studies, No. 31, Princeton Univ. Press, Princeton, N. J., 1953.

5. T. Naito, On a problem of Wolk in interval topologies, Proc. Amer. Math. Soc. 11 (1960), 156-158.

6. E. S. Wolk, Order-compatible topologies on a partially ordered set, Proc. Amer. Math. Soc. 9 (1958), 524-529.

7. - On partially ordered sets possessing a unique order-compatible topology, Proc. Amer. Math. Soc. 11 (1960), 487-492.

8. - Dedekind completeness and a fixed point theorem, Canad. J. Math. 9 (1957), 400-405.

UNIVERSITY OF CoNNECTICUT AND

UNIVERSITY OF ROCHESTER 